\title{
Diseño y construcción de un prototipo para la medición de distorsión armónica en redes eléctricas
}

\section{Designing and building a harmonic-distorsion measurement prototype for electrical networks}

\section{Duvan Cardona}

Tecnólogo en electricidad. Tecnólogo de Condensa SA Esp. Bogotá. Colombia. Contacto:dfcardonaa@correo.udistrital.edu.co

\section{DANILO LÓPEZ}

Ingeniero Electrónico. Docente de la Universidad Distrital Francisco José de Caldas. Bogotá, Colombia. Contacto:dalopezs@udistrital.edu.co

\section{César A. Hernández}

Ingeniero Electrónico. Docente de la Universidad Distrital Francisco José de Caldas. Bogotá, Colombia. Contacto: cahernandezs@udistrital.edu.co

Fecha de recepción: 27 de marzo de 2012

Clasificación del artículo: Revisión de Tema

Fecha de aceptación: 27 de noviembre de 2012

Financiamiento: Universidad Distrital

Palabras clave: algoritmo, diezmado, factor de giro, FFT, THD.

Key words: algorithm, decimated, twist factor, FFT, THD.

\section{RESUMEN}

El presente artículo ilustra la forma de implementar la transformada rápida de Fourier usando el algoritmo de Cooley-Tukey, aplicado al análisis y el cálculo de la distorsión armónica en hogares y pequeñas empresas donde no se tiene la certeza de la calidad de energía que les es entregada a diario. La investigación está basada en una arquitectura de microcontrolador para obtener un buen rendimiento con un lenguaje de alto nivel, además, se desarrollan las diferentes partes del hardware necesarias para el cálculo de la FFT de 1024 puntos y el cálculo del nivel de THD.

\begin{abstract}
This investigation work shows the way to implement the rapidly transformed of Fourier using the algorithms of Cooley-Tukey, applied to the analysis and calculation of the harmonic distortion in homes and small businesses that it's don't knows the energy's quality that is
\end{abstract}


delivered daily. The investigation is based on a microcontroller architecture for good perfomance whit a high level language, olso develop the diferent parts of hardware necesary for the reckoning of the FFT of 1024 points and the reckoning of THD level.

\section{INTRODUCCIÓN}

La calidad de servicio es un tema que preocupa tanto a empresas eléctricas como a consumidores, ya que las perturbaciones en una red pueden alterar el correcto funcionamiento de los equipos instalados. Durante la última década, se han desarrollado muchas aplicaciones informáticas para simular y analizar problemas de calidad de servicio, el aumento de equipos sensibles a variaciones de tensiones y corrientes, como computadoras, o equipos de alta precisión, ha incrementado la preocupación de empresas eléctricas y consumidores por la calidad del servicio.

El mal funcionamiento de los equipos en redes de potencia puede ser originado por un amplio espectro de perturbaciones que pueden ser originadas tanto en redes de potencia como en las instalaciones de los usuarios.

El avance tecnológico, en cuanto a plataformas robustas, ha permitido la implementación de diferentes algoritmos que permiten técnicas de simulaciones digitales, las cuales han sido métodos muy eficaces en el estudio de la calidad del servicio, estas técnicas están basadas en el empleo de transformadas [1]. Una de las técnicas utilizadas para este análisis es la transformada rápida de Fourier (FFT), para la cual se han implementado diversos algoritmos, la FFT transforma una señal discreta en el dominio del tiempo a su representación en el dominio de la frecuencia, de una manera rápida y eficiente, y se convierte en un método muy sencillo en el análisis de las perturbaciones que presenta la red [2], [3].
El algoritmo aquí utilizado es el de CooleyTukey, ya que es el algoritmo base para el cálculo de la FFT por ser el primer algoritmo realizado, y permite realizar modificaciones que optimizan el cálculo este algoritmo, este algoritmo consiste básicamente en dividir por dos la cantidad de datos discretos que se tienen de una señal e ir separándolos hasta obtener $\mathrm{n}$ cantidad de parejas de datos [4].

\section{METODOLOGÍA}

Para realizar este proyecto se utilizó un microcontrolador 18f2550, también el conversor ADC (convertidor analógico digital) del microcontrolador para discretizar la señal, para el cálculo de la FFT se implementó el método de Cooley-Tukey con una modificación, partiendo del hecho que se tiene una señal dada polinomialmente [1] como la ecuación (1):

$$
X(k)=\sum_{n=0}^{N-1} x[n] W_{N}^{k \cdot n}
$$

Siendo:

$\mathrm{N}$ el número de datos.

n la posición del dato.

W el factor de giro.

$\mathrm{k}$ constante multiplicativa

Sabiendo que la cantidad de datos $\mathrm{N}$ es par potencia de 2, se empieza a descomponer la sumatoria, dividiéndola en 2 de la siguiente manera, ecuación (2): 


$$
\begin{gathered}
X(k)=\sum_{n=0}^{N / 2-1} x[2 n] W_{N}^{k .2 n}+ \\
\sum_{n=0}^{N / 2-1} x[2 n+1] W_{N}^{k \cdot(2 n+1)}
\end{gathered}
$$

Reorganizando la ecuación se reemplaza $x[2 n]$ por $\mathrm{f}[\mathrm{n}]$ y $\mathrm{x}[2 \mathrm{n}+1]$ por $\mathrm{g}[\mathrm{n}]$ y se obtienen las ecuaciones (3) y (4).

$$
\begin{aligned}
& F(k)=\sum_{n=0}^{N / 2-1} f[n] W_{N / 2}^{k \cdot n} \\
& G(k)=\sum_{n=0}^{N / 2-1} g[n] W_{N / 2}^{k . n}
\end{aligned}
$$

Siendo $F(k)$ y $G(k)$ funciones periódicas, se puede ir reduciendo en N/2 la longitud de cada sumatoria hasta obtener $\mathrm{n}$ sumatorias de longitud 2 , siendo esta la unidad básica de la FFT conocida como mariposa, ecuación (5), ecuación (6) y que se explica más adelante.

$$
\begin{gathered}
F\left(k+\frac{N}{2}\right)=\sum_{n}^{N / 2-1} f(n) W_{\underline{N}}^{\left(k+\frac{N}{2}\right) \cdot n}= \\
\sum_{n}^{N / 2-1} f(n) W_{N / 2}^{k n} \cdot e^{-j \frac{2 \pi}{N / 2}(N / 2) n}=F(K) \\
F(k)=\sum_{n}^{N / 4-1} f_{f}(n) W_{N / 2}^{k 2 n}+ \\
W_{N / 2}^{k} \cdot \sum_{n}^{N / 4-1} g_{f}(n) W_{N / 2}^{k 2 n}
\end{gathered}
$$

Donde y son lo mismo para $\mathrm{f}[\mathrm{n}]$ que para $\mathrm{x}[\mathrm{n}]$. Analógicamente, se hace los mismo con $\mathrm{G}(\mathrm{k})$. Es decir, que ahora sobre $\mathrm{f}[\mathrm{n}]$ y $\mathrm{g}[\mathrm{n}]$ se realizan 4 DFT de longitud N/4. Entonces, se pueden hacer múltiples divisiones del intervalo $[0, \mathrm{~N}-1]$ mientras se pueda dividir $\mathrm{N}$ entre 2 .

Finalmente, se se utilizó una display LCD de 16x2 para la visualización de los resultados.

\subsection{Conversor analógico digital}

Una vez se tiene la señal de tensión, se inicia la función del ADC del microcontrolador y se procede a tomar lectura de la señal, por cada flanco de subida de una señal de reloj, cuyo periodo es 31,14 micro segundos, se toma una lectura para asi tomar los 1024 datos en un periodo de la señal que corresponde a 16,66 mili segundos, a su vez, estos datos se van almacenando en un arreglo previamente declarado en la memoria ROM (memoria de solo lectura no volátil) del microcontrolador [5], [6].

\subsection{Algoritmo para obtener la FFT}

En primera instancia se verificó la efectividad de la FFT por medio de un software avanzado (Matlab) para el cálculo de la transformada discreta de Fourier, que es implementada para representar una señal en el dominio de la frecuencia [7].

El algoritmo que ha sido utilizado para la obtención de la FFT (cooley tukey modificado) se describe a continuación: primero, se realizó una réplica del algoritmo de cooley tukey (modificado) mencionado anteriormente, y se le aplicó a una cadena de datos, con una precisión de tan solo 4 bits como prueba piloto, para realizar este algoritmo la cadena de datos tiene que tener una cantidad de datos igual a $\mathbf{N}$, siendo $\mathbf{N}$ una potencia de 2 .

La implementación de la réplica de este algoritmo se realizó de la siguiente manera: en primer lugar, se hizo un diezmado en el dominio del tiempo de los datos a analizar, el diezmado consiste en tomar la cadena de datos que se tiene y separar los datos que se encuentran ubicados en las posiciones pares de la cadena con los de las posiciones impares de la misma, obteniendo así dos cadenas de datos con una longitud igual a la mitad de la longitud inicial de la cadena principal, este proceso se repite hasta obtener $\mathrm{n}$ cadenas de datos con una longitud igual a 2 , siendo esta, la unidad básica de la FFT conocida como la mariposa, en donde sólo se necesita una multiplicación y dos sumas complejas.

El cálculo de la FFT se realiza en un número de etapas equivalente al logaritmo en base 2 del 
número de puntos $\mathbf{N}$, donde, en cada etapa, la salida es la entrada de la siguiente, es decir; en el ejemplo piloto se tienen 4 bits (16 datos); en la primera etapa, los datos se almacenan en un primer arreglo de datos en la memoria ROM del microcontrolador, estos datos son la entrada de la segunda etapa donde se hace el primer diezmado, en la salida de esta etapa se tienen dos cadenas de datos cada una de ocho datos, que se almacenan en un segundo arreglo en la memoria ROM del microcontrolador; a su vez, esta salida se convierte en la entrada de la tercera etapa donde se hace nuevamente un diezmado de datos $y$, en la salida de esta etapa, se obtienen cuatro cadenas de cuatro datos, que se almacenan en el primer arreglo anteriormente utilizado; por último, la salida de esta etapa, se convierte en la entrada de la cuarta etapa donde se realiza el último diezmado, donde se obtiene en la salida de esta etapa ocho cadenas de dos datos, almacenándose en el segundo arreglo que se utilizó anteriormente, en la figura 1 se observan las etapas realizadas para la obtención de la FFT de 16 puntas [8], [9].

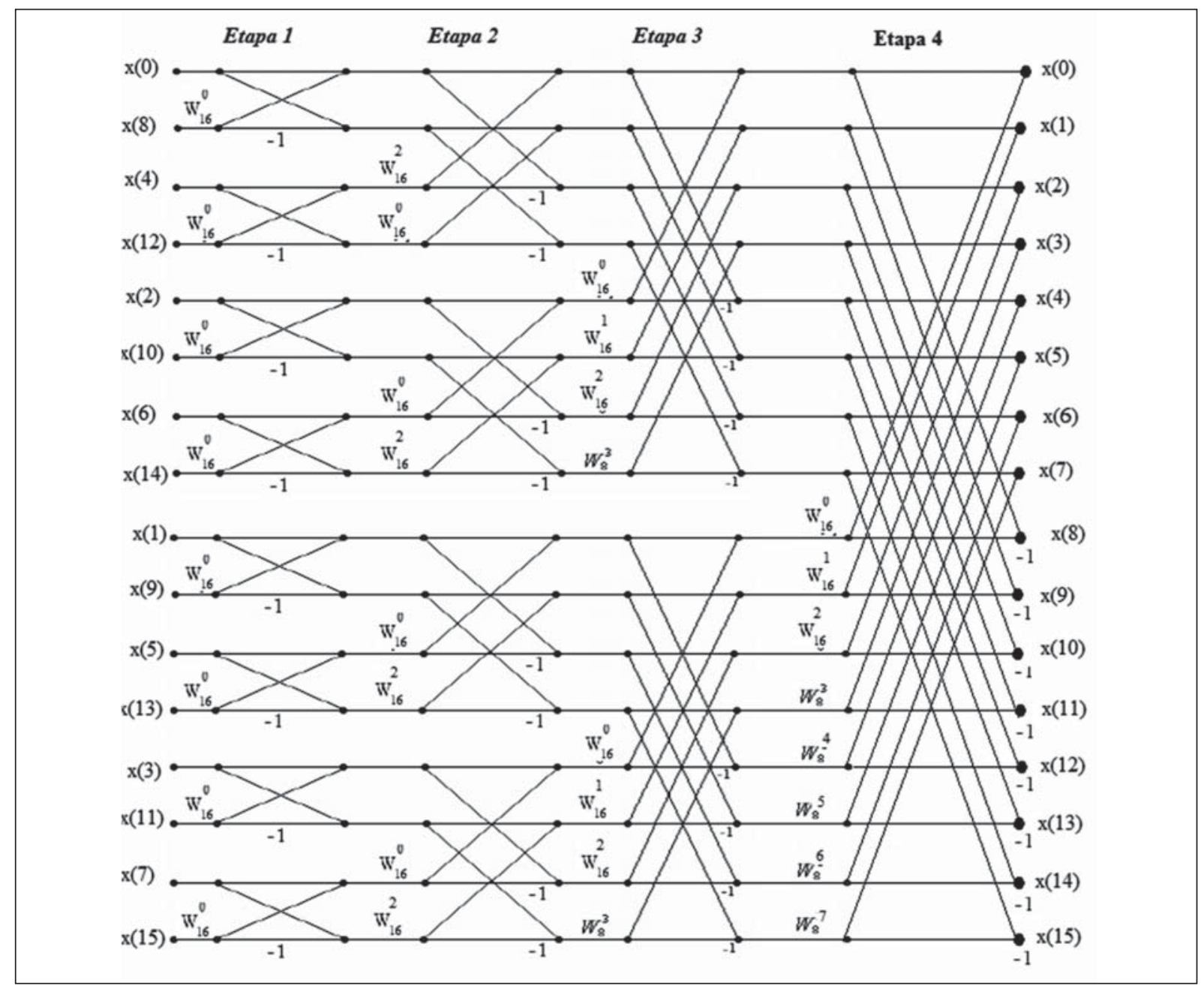

Figura 1. Diagrama de flujo para un algoritmo FFT de 16 puntos

Fuente: elaboración propia. 
En la figura 1 es el factor de giro, $\mathrm{N}$ es el número de datos, a es las veces que se ha diezmado y $n$ es la posición del dato en el arreglo.

Posteriormente, se procedió a multiplicar por un factor de giro o constante multiplicativa, ecuación (7), correspondiente a cada dato en cada etapa, según la cantidad de diezmados que se le hayan aplicado a cada dato. Para explicar un poco mejor esto, se tienen las siguientes ecuaciones:

$$
X(k)=\sum_{n=0}^{N-1} x[n] W_{N}^{k . n}
$$

Diezmado de los datos, separando los que están ubicados en las posiciones pares de las impares, siendo $\mathrm{X}[\mathrm{n}]$ el dato en la posición $\mathbf{n}$ se tiene la ecuación (8).

$$
\begin{gathered}
X(k)=\left(X[0] W_{\frac{N}{2}}^{0 . K}+X[2] W_{\frac{N}{2}}^{1 . K}\right)+ \\
\left(X[1] W_{\frac{N}{2}}^{0 . K}+X[1] W_{\frac{N}{2}}^{1 . K}\right) \cdot W_{N}^{K}
\end{gathered}
$$

Reemplazando W (Factor de giro) por la constante de Euler correspondiente para cada dato la ecuación (9).

$$
W_{N}^{K}=e^{-i \frac{2 \pi}{N} \cdot 2 K}=\cos \left(\frac{2 . K . \pi}{\frac{N}{2}}\right)-i \sin \left(\frac{2 . K \cdot \pi}{\frac{N}{2}}\right)
$$

Posteriormente, se compararon los datos obtenidos con la aplicación de este algoritmo, con los datos que arroja el software Matlab (software de alto nivel), una vez realizadas las principales pruebas se inicia la extensión del código realizado a una capacidad de 10 bits (1024 puntos). Para proceder con la FFT de 10 bits (1024 puntos), se escogió un micro de la familia $18 \mathrm{fxx}$, para almacenar los datos discretizados se reservó un arreglo de 1024 posiciones de 32 bits, posteriormente, para diezmar los datos, se reservaron dos arreglos más de 32 bits utilizando punteros para un mejor aprovechamiento de la memoria ROM del micro.

El diseño del algoritmo se divide en cuatro bloques funcionales distribuidos como se indica en

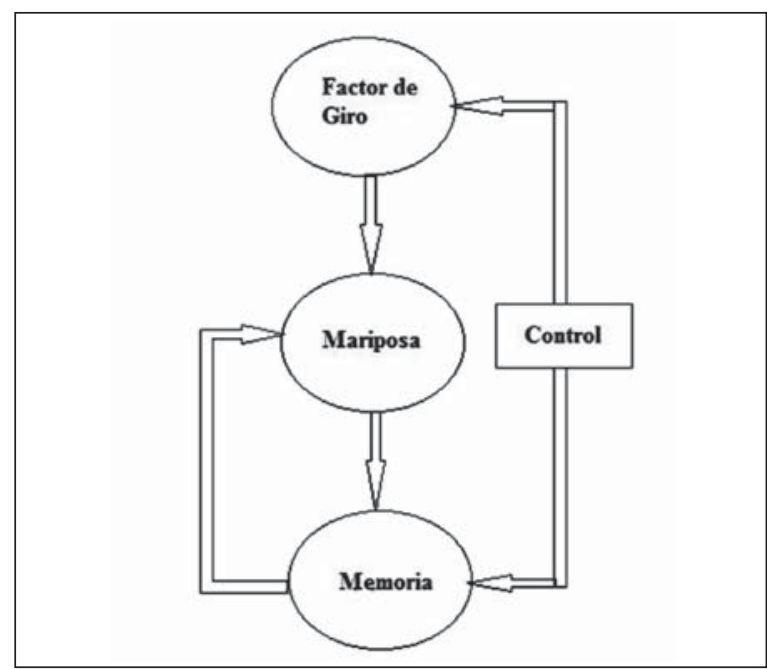

Figura 2. Diagrama de bloques del algoritmo Fuente: elaboración propia.

la figrua 2, que realizan el proceso del cálculo sucesivo de la FFT de 2 puntos para las muestras almacenadas en el arreglo principal, los datos procesados ocupan un tamaño de 32 bits para la componente real y 32 bits para la componente imaginaria, la parte de la mariposa procesa los datos de entrada procedentes de la memoria, con los factores de giro, determinantes por el exponente calculado en el bloque de control, y entrega el resultado de nuevo para ser procesado en la siguiente etapa. Ver figura 2.

Una vez los datos ingresados, provenientes del conversor analógico digital, se almacenaron en un rango de memoria de $1024 \times 32$, se procedió con el almacenamiento de los datos procesados por medio de dos rangos de memoria más de 1024x32; una vez se tienen estos datos totalmente procesados, se les aplicó el factor de giro y se almacenaron en dos nuevos rangos de memoria de $1024 \times 34$, uno para la parte real y otro para la parte imaginaria. La necesidad de usar dos rangos de memoria para el procesamiento de datos se da ya que es necesario una vez guardados los datos leerlos nuevamente para así procesarlos nuevamente, esto se ilustra mejor en la tabla 1. 
Tabla 1. Escritura y lectura de memoria en los rangos establecidos, según la etapa

\begin{tabular}{|l|l|l|l|}
\hline Etapas de procesamiento & \multicolumn{1}{|c|}{$\mathbf{1}$} & \multicolumn{1}{|c|}{$\mathbf{2}$} & \multicolumn{1}{|c|}{$\mathbf{3}$} \\
\hline Lectura & Lee en los datos originales & $\begin{array}{l}\text { Lee en el primer rango de } \\
\text { memoria }\end{array}$ & $\begin{array}{l}\text { Lee en el segundo rango de } \\
\text { memoria }\end{array}$ \\
\hline Escritura & $\begin{array}{l}\text { Escribe en el primer rango de } \\
\text { memoria }\end{array}$ & $\begin{array}{l}\text { Escribe en el segundo rango } \\
\text { de memoria }\end{array}$ & $\begin{array}{l}\text { Escribe en el tercer rango de } \\
\text { memoria }\end{array}$ \\
\hline
\end{tabular}

Fuente: elaboración propia.

Los factores de giro se almacenaron cada uno en un espacio de memoria de 32 bits, estos factores se obtuvieron multiplicando unos valores preestablecidos por una constante $\mathrm{k}$, que varía de acuerdo al índice de cada etapa, estos factores de giro están representados por 128 configuraciones de bits.

\section{RESULTADOS}

A continuación se describen los resultados obtenidos en la presente investigación en términos de los tiempos de procesamiento, la precisión y eficacia del algoritmo modificado e implementado y la eficiencia de este.

\subsection{Tiempos de procesamiento}

Luego de realizar simulaciones, resulta prometedor el desempeño del procesador FFT que se ha diseñado. Inicialmente se cuenta con un oscilador de $40 \mathrm{MHz}$, lo que lleva al sistema a hacer un cálculo completo de la FFT de 1024 puntas en $44,69 \mathrm{~ms}$, para un total de 45826,81 transformadas por segundo.

Se utilizaron pruebas en Matlab que confirman la efectividad del algoritmo y los procesos usados en el microcontrolador.

En la figura 3 se puede observar la velocidad de procesamiento de la FFT con distintas transfor-

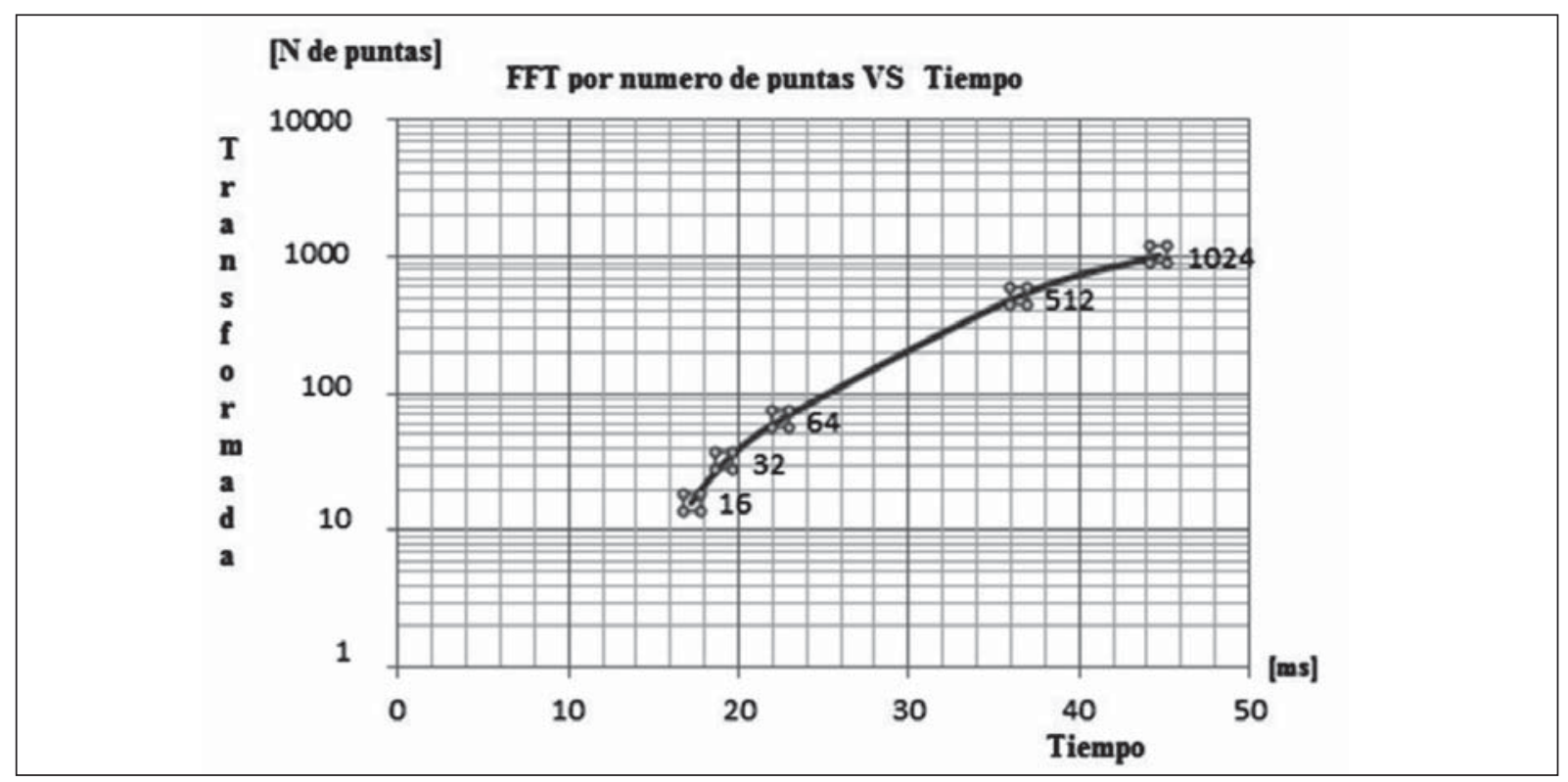

Figura 3. FFT de distintas puntas vs. tiempo

Fuente: elaboración propia. 
Tabla 2. Comparación entre Matlab y la FFT con una muestra de 16 datos

\begin{tabular}{|c|c|c|c|}
\hline \multicolumn{4}{|c|}{ Transformada de 16 puntos } \\
\hline Matlab & FFT & Error absoluto & Error relativo \\
\hline 136 & 136 & 0 & $0 \%$ \\
\hline$-8+40,22 i$ & $-7,99+40,21 i$ & 0,017 & $0,041 \%$ \\
\hline$-8+19,34 i$ & $-7,99+19,31 i$ & 0,03 & $0,14 \%$ \\
\hline$-8+11,97 i$ & $-7,99+11,97 i$ & 0,001 & $0,0069 \%$ \\
\hline$-8+8 i$ & $-7,99+8 i$ & 0,01 & $0,088 \%$ \\
\hline$-8+5,35 i$ & $-7,99+5,34 i$ & 0,01 & $0,1039 \%$ \\
\hline$-8+3,31 i$ & $-7,99+3,31$ & 0,01 & $0,115 \%$ \\
\hline$-8+1,59 i$ & $-7,99+1,59 i$ & 0,01 & $0,122 \%$ \\
\hline-8 & -8 & 0 & $0 \%$ \\
\hline$-8-1,59 i$ & $-8-1,59 i$ & 0 & $0 \%$ \\
\hline$-8-3,31 i$ & $-8-3,31 i$ & 0 & $0 \%$ \\
\hline$-8-5,35 i$ & $-8-5,34 i$ & 0 & $0 \%$ \\
\hline$-8-8 i$ & $-8-7,99 i$ & 0 & $0 \%$ \\
\hline$-8-11,97 i$ & $-8-11,97 i$ & 0 & $0 \%$ \\
\hline$-8-19,31 i$ & $-8-19,31 i$ & 0 & $0 \%$ \\
\hline$-8-40,22 i$ & $-8-40,21 \mathrm{i}$ & 0,017 & $0,041 \%$ \\
\hline
\end{tabular}

Fuente: elaboración propia.

madas (16 puntas, 32 puntas, 64 puntas y 1024 puntas).

\subsection{Precisión del algoritmo}

Los resultados que se obtuvieron en cuanto a precisión al comparar el proceso realizado por el microcontrolador frente al proceso que realiza Matlab se muestran a continuación en la tabla 2 .

Sin embargo, tomando todos los datos de la tabla anterior, se obtiene un error relativo total de $0,41 \%$.

\subsection{Eficiencia del algoritmo}

Al hacer el cálculo de la cantidad de operaciones complejas que se requieren para el obtener de la transformada, se confirma la eficiencia del algoritmo utilizado ver tabla 3.
Tabla 3. Número de operaciones para la transformada discreta y el algoritmo utilizado

\begin{tabular}{|c|c|c|}
\hline$N^{\circ}$ de puntos & $\begin{array}{c}\text { Algoritmo } \\
\text { utilizado }\end{array}$ & $\begin{array}{c}\text { Transformada } \\
\text { Discreta }\end{array}$ \\
\hline 2 & 2 & 4 \\
\hline 8 & 24 & 64 \\
\hline 32 & 160 & 1024 \\
\hline 64 & 384 & 4096 \\
\hline 256 & 2048 & 65536 \\
\hline 512 & 4608 & 262144 \\
\hline 1024 & 10240 & 1048576 \\
\hline
\end{tabular}

Fuente: elaboración propia.

Con la figura 4 se observó el crecimiento en cuanto a la cantidad de operaciones complejas tanto en el algoritmo utilizado como en la transformada de Fourier Discreta. 


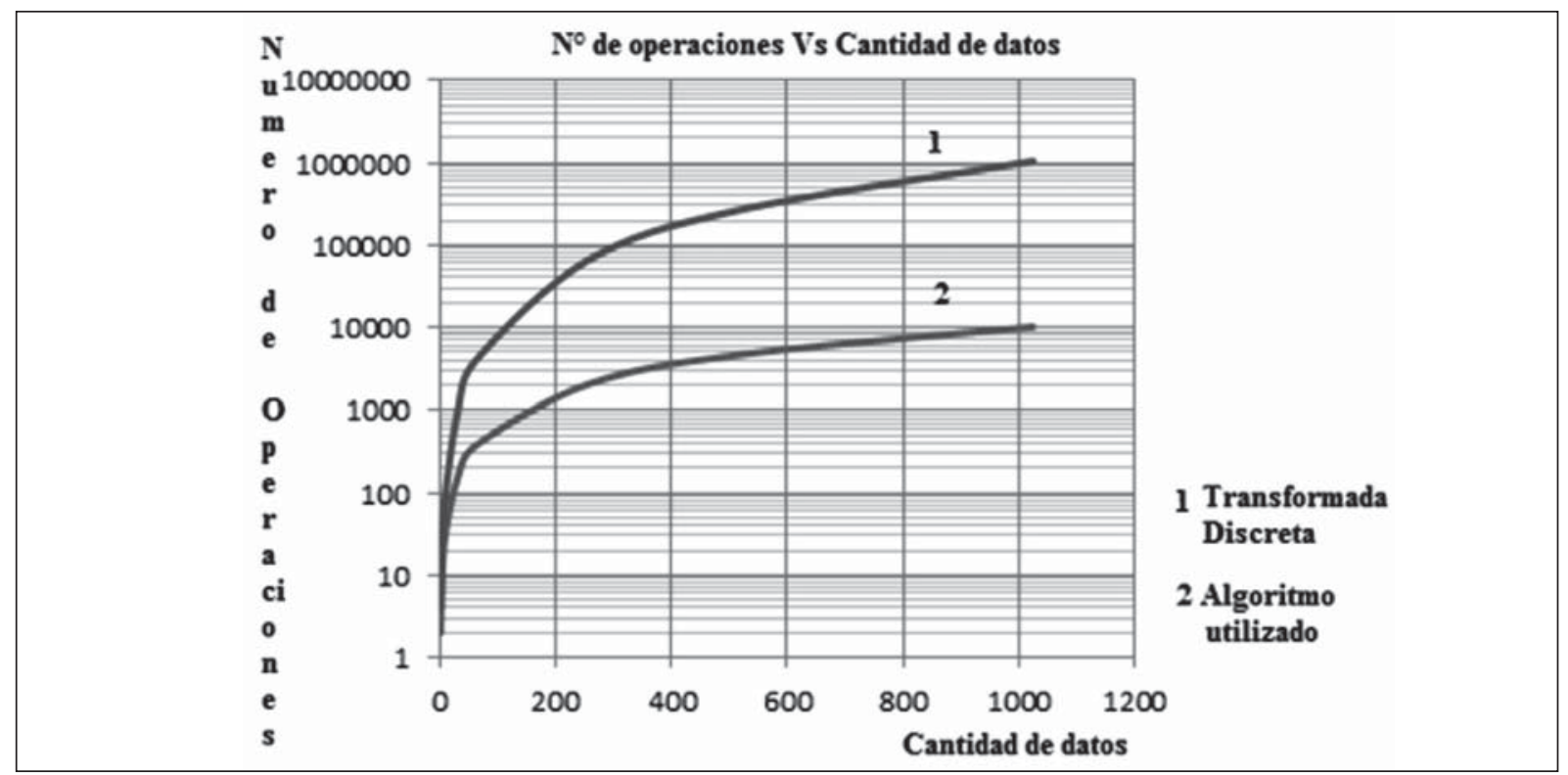

Figura 4. Cantidad de operaciones complejas utilizadas

Fuente: elaboración propia.

Analizando el tiempo en que el microcontrolador lee los datos entregados por el ADC, se comparó con el tiempo en que el microcontrolador tarda en entregar los datos transformados, y así se obtuvo un promedio de datos leídos por segundo y datos entregados por segundo para posteriormente calcular la eficiencia del algoritmo en porcentaje. Ver tabla 4.

Tabla 4. Eficiencia del algoritmo

\begin{tabular}{|c|c|c|}
\hline $\begin{array}{c}\mathrm{N} \text { de datos de } \\
\text { entrada }\end{array}$ & $\begin{array}{c}\text { Tiempo en que se } \\
\text { toman los datos }\end{array}$ & $\begin{array}{c}\mathrm{N} \text { de datos por } \\
\text { segundo }\end{array}$ \\
\hline 1042 & $16,66[\mathrm{~ms}]$ & $62545,02[\mathrm{~d} / \mathrm{s}]$ \\
\hline $\begin{array}{c}\mathrm{N} \text { de datos de } \\
\text { salida }\end{array}$ & $\begin{array}{c}\text { Tiempo de proce- } \\
\text { samiento }\end{array}$ & $\begin{array}{c}\mathrm{N} \text { de datos por } \\
\text { segundo }\end{array}$ \\
\hline 2048 & $44,69[\mathrm{~ms}]$ & $45826,81[\mathrm{~d} / \mathrm{s}]$ \\
\hline & Eficiencia & $73,27 \%$ \\
\hline
\end{tabular}

Fuente: elaboración propia.

\section{CONCLUCIONES}

La arquitectura de esta FFT cooley tukey modificado brinda varios beneficios, como: bajo número de funciones combinacionales, registros y memoria en comparación con otros trabajos realizados.

La metodología aplicada en el diseño del código para el presente artículo permite una fácil configuración del sistema para $\mathrm{N}=$ datos de entrada $(\mathrm{n} \geq 2)$, de cualquier número de bits. Esto se logra debido a la creación de bloques funcionales que permiten una fácil manipulación de los datos. Por lo tanto, se ha obtenido una arquitectura base que puede utilizarse en varias situaciones para diferentes requerimientos.

\section{FINANCIAMIENTO}

El presente trabajo hace parte del proyecto de investigación "Diseño y construcción de un prototipo de medición de los indicadores de calidad del servicio de la energía eléctrica para usuario residencial", financiado por el CIDC. 


\section{Referencias}

[1] M. Jamali, J. Downey, N. Wilikins, C. Rehm and J. Tipping, "Development of a fpga-based high speed fft processor for wideband direction of arrival applications", Radar Conference, 2009 IEEE, May, 2009, pp. 1-4.

[2] J. Proakis, Digital Signal Processing: Principles, Algorithms, and Applications. Englewood Cliffs, NJ: Prentice-Hall, 1996.

[3] Oppenheim, R. Schaefer and J. Buck, Discrete-Time Signal Processing. Englewood Cliffs, NJ: Prentice-Hall, 1998.

[4] J. Cooley and J. Tukey, "An algorithm for the machine calculation of complex fourier series", Math. Comput., Vol. 19, pp. 297301, Apr 1965.

[5] H. Sorensen, M. Heideman and C. Burrus, "On computing the split-radix fft" Acoustics, Speech and Signal Processing, IEEE
Transactions on, Vol. 34, No. 1, pp. 152156, Feb, 1986.

[6] J. Kuo, C. Wen, C. Lin and A. Wu, "VLSI design of a variable length FFT/IFFT processor for OFDM based communication systems", J. Appl. Signal Process., Vol. 13, pp. 1306-1316, Jan. 2003.

[7] X. Guan, Y. Fei and H. Lin, "A hierarchical design of an application specific instruction set processor for high-throughput FFT", Proc. Int. Symp. Circuits Syst., May, 2009, pp. 2513-2516.

[8] H. Ishebabi, G. Ascheid, H. Meyr, O. Atak, A. Atalar and E. Arikan, "An efficient parallelization technique for high throughput FFT-ASIPs" in Proc. Int. Symp. Circuits Syst., May 2006, pp. 5664-5667.

[9] G. Liu and Q. Feng, "ASIC design of lowpower reconfigurable FFT processor", in Proc. Int. Conf. ASIC, Oct. 2007, pp. 44-47. 